\title{
MERALGIA PARAESTHETICA OF SPINAL ORIGIN: BRIEF REPORT
}

\author{
JIANG GUO-XIANG, XU WEI-DONG
}

Meralgia paraesthetica is usually thought of as an entrapment syndrome in the region of the inguinal ligament. We have shown, however, that it may originate in the spine, at the L3/4 level (Jiang Guo-Xiang, Xu WeiDong and Wang Ai-Hao 1988). On this basis we have treated 14 patients successfully by an epidural injection of dexamethasone.

Patients and Method. We have treated 14 patients with 15 affected limbs. All were men; the median age was 44 . All the patients complained of intermittent hypoaesthesia on the lateral aspect of the thigh, chiefly when walking; some also complained of tiredness. The area

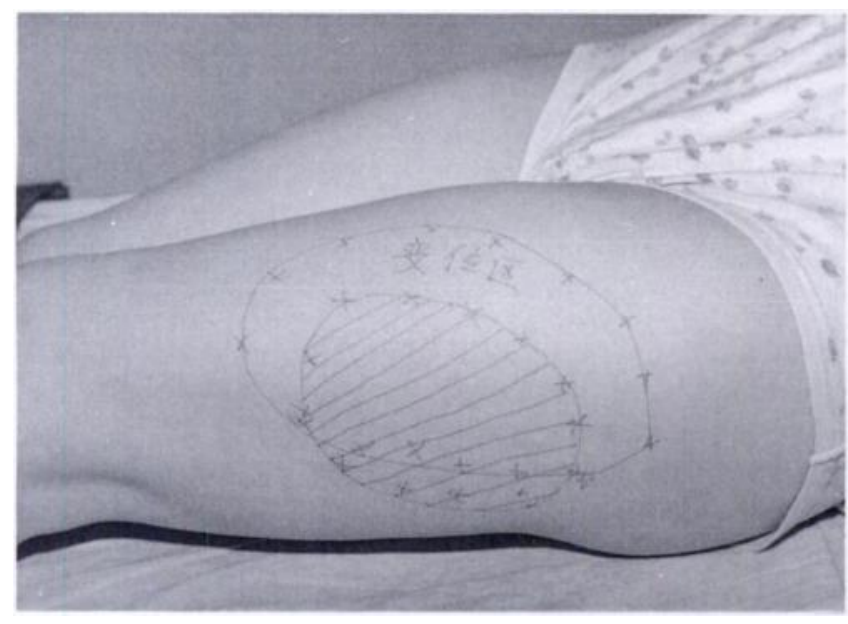

Fig. 1

affected was marked and the effect of treatment was assessed by comparing the area affected before with that after injecting dexamethasone, using transparent film.

Two special tests were used which also confirmed that the meralgia was of spinal origin. 1) The position changing test. The patient lies supine and the hypoaesthetic area is marked and measured; a pillow which hyperextends the lumbar spine is placed in position and, after a few minutes, the area is measured again. An increase (Fig 1) denotes a positive test. 2) The backward stretch test. The standing patient stretches backwards

Jiang Guo-Xiang, Visiting Surgeon and President

$\mathrm{Xu}$ Wei-Dong, Resident Surgeon

Railway Central Hospital, Urumchi, China.

Correspondence should be sent to Dr Jiang Guo-Xiang.

(C) 1988 British Editorial Society of Bone and Joint Surgery $0301-620 X / 88 / 5 R 06 \$ 2.00$

J Bone Joint Surg [Br] 1988;70-B:843-4. for 15 or 20 seconds; the test is positive if this causes any increase of symptoms. These two tests were positive in all our patients.

Table I. Effects of epidural injections for meralgia paresthetica

\begin{tabular}{|c|c|c|c|}
\hline \multirow[b]{2}{*}{$\begin{array}{l}\text { Case } \\
\text { number }\end{array}$} & \multicolumn{3}{|c|}{ Affected area in $\mathrm{cm}^{2}$} \\
\hline & $\begin{array}{l}\text { Before } \\
\text { injection }\end{array}$ & $\begin{array}{l}\text { Soon after } \\
\text { injection }\end{array}$ & At follow-up \\
\hline 1 & 560 & 4.0 & 0 \\
\hline 2 & 160 & $\mathbf{0}$ & $\mathbf{0}$ \\
\hline 3 & 427 & $\mathbf{0}$ & $\mathbf{0}$ \\
\hline 4 & 84 & 23.5 & 0 \\
\hline 5 & 276 & 25.0 & 74 \\
\hline 6 & 220 & 3.0 & 0 \\
\hline 7 & 482 & 0 & 0 \\
\hline 8 & 254 & 36.0 & 0 \\
\hline 9 & 102 & 60.0 & 98 \\
\hline 10 & 101 & 17.5 & 0 \\
\hline 11 & 320 & 0 & 0 \\
\hline 12 & 54 & 24.0 & 53 \\
\hline 13 & 75 & 0 & 0 \\
\hline 14 & 239 & 0 & 80 \\
\hline 15 & 352 & 5.0 & 104 \\
\hline
\end{tabular}

The epidural injection is performed ideally between the spinous processes of $\mathrm{L} 3$ and L4, but in case of difficulty one space higher or one lower was used. The skin is cleaned with iodine and alcohol and $2 \mathrm{ml}$ of a 1 or $2 \%$ procaine solution injected through a No. 7 needle. Then the point of a No. 16 epidural needle attached to a $2 \mathrm{ml}$ syringe is used to probe for the epidural space. When it reaches the ligamentum flavum resistance is so great that nothing can be injected, but when it enters the epidural space the fluid flows freely. Having checked that the needle is in the epidural space (and not inside the dura) the dexamethasone is injected and the patient remains on the couch for 15 minutes before returning to the ward. During the injection the patient often feels a sensation of coolness in the lumbar spine and lower limbs. With too rapid an injection there may be pain.

A single dose consists of $10 \mathrm{mg}$ dexamethasone mixed with either 0.25 to $1 \%$ procaine or $0.9 \%$ saline to a total volume of 10 to $15 \mathrm{ml}$. A single injection may be 
sufficient to relieve symptoms, but three or four injections at weekly intervals may be needed.

Results. These are shown in Table I. It is clear that in all cases the affected area is considerably reduced immediately after the injection and in all but two the effect persisted at follow-up, which was at 10 to 32 months (average 18 months). In 10 cases the area affected had been reduced to zero.

Discussion. Many surgeons still base their treatment of meralgia paraesthetica on the assumption that the causal lesion is a local one in the region of the inguinal ligament (Jones 1974). This assumption does not explain the association with spinal stenosis, nor the two tests we have described, nor the occasional bilateral case. Moreover, the considerable success obtained by epidural injection seems to us to justify the conclusion that, at least in some cases, the causal lesion is at $\mathrm{L} 3 / 4$.

\section{REFERENCES}

Jiang G-X, Xu W-D, Wang A-H. Spinal stenosis with meralgia paresthetica. J Bone Joint Surg [Br] 1988;70-B:272-3.

Jones RK. Meralgia paresthetica as a cause of leg discomfort. Can Med Assoc J 1974;111:541-2.

\title{
THE FLEXION SIGN OF NEEDLE PLACEMENT FOR HIP ARTHROGRAPHY: BRIEF REPORT
}

\author{
ANTHONY A. BERNARD
}

Accurate needle placement is important in arthrography of the neonatal hip, but although various methods have been described, difficulties may occur. Ease of injection (Mitchell 1963) and re-aspiration of saline may be misleading, since they may be seen after extracapsular injection. The injection of a small amount of air (Crawford and Carothers 1982) or contrast medium, has been described but this may tend to obscure the subsequent images. There is also a small risk of air embolism (McCauley, Wunderlich and Zimbler 1981). Aspiration of synovial fluid or joint contents is only effective when the joint contains sufficient fluid. A "waggle" sign and suction air arthrography have recently been described (Lancaster and Cummings 1987), but this deviation of the needle is caused by hip rotation, and carries some danger of intra-articular damage.

Technique. The technique described in this paper was used for hip arthrography in 60 cases, 42 of them for congenital dislocation. The child is anaesthetised, placed supine on a radiolucent table and the area cleaned and draped. A short-bevelled, 18-gauge spinal needle with the stylet in place is inserted, in the neonate, two fingerbreadths distal and two fingerbreadths medial to the anterior superior iliac spine. The femoral artery is palpated and the tip of the needle is directed deep to it into the anterior aspect of the capsule of the hip. A giving-way sensation is usually felt as the needle punctures the capsule.

A fluoroscopic check is made to ensure that the tip of the needle is opposite the middle of the femoral neck

A. A. Bernard, MD, FRCS Ed, Late Registrar, The Royal Orthopaedic Hospital, Birmingham.

Department of Orthopaedics, Saint Luke's Hospital, Guardamangia, Malta.

(C) 1988 British Editorial Society of Bone and Joint Surgery

0301-620X/88/5R $78 \$ 2.00$

J Bone Joint Surg [Br] 1988;70-B:844. and not in the region of the limbus, where clear visualisation is needed. The stylet is then removed and 5 to $10 \mathrm{ml}$ of saline are injected from a $20 \mathrm{ml}$ syringe. As the joint is distended, the lower limb is seen to move into a few degrees of flexion and lateral rotation. When the syringe is removed from the hub of the needle, saline will squirt out under pressure as the limb returns to its original position. Injection of contrast medium is then carried out.

Discussion. The flexion and lateral rotation of the limb produced by stretching of the capsule on distension are caused by the orientation of its fibres in a spiral fashion. Distension of the hip causes it to move into a position that maximises the joint capacity by "unwinding" the capsule.

The flexion sign was seen to be positive in all normal or subluxated hips, but was less marked in grossly displaced hips. In these cases the diagnosis was clinically obvious, and placement of the needle into the empty acetabulum was easily accomplished. No complications have arisen from this simple method of ensuring accurate placement of the needle.

No benefits in any form have been received or will be received from a commercial party related directly or indirectly to the subject of this article.

\section{REFERENCES}

Crawford AH, Carothers TA. Hip arthrography in the skeletally immature. Clin Orthop 1982;162:54-60.

Lancaster SJ, Cummings RJ. Hip aspiration: verification of needle position by air arthrography. J Pediatr Orthop 1987;7:91-2.

McCauley RGK, Wunderlich BK, Zimbler S. Air embolism as a complication of hip arthrography. Sketal Radiol 1981;6:11-3.

Mitchell GP. Arthrography in congenital displacement of the hip. $J$ Bone Joint Surg [Br] 1963;45-B:88-95. 